\title{
Detection of the Electromagnetic Equivalents of the Emotional Characteristics of Words: Implications for the Electronic-Listening Generation
}

\author{
Kevin S. Saroka ${ }^{1,2}$ and Michael A. Persinger ${ }^{*}, 1,2,3$ \\ Behavioural Neuroscience Laboratory ${ }^{1}$, Departments of Psychology ${ }^{2}$ and Biology ${ }^{3}$, Laurentian University, Sudbury, \\ Ontario, P3E 2C6, Canada
}

\begin{abstract}
The audible energies associated with intense meaningful words significantly affect our experience and responses. Personal listening devices (such as music players) emit weak-intensity magnetic fields, that are shadowed by the sonic patterns, which have been associated with the induction of affective experiences experimentally within the laboratory. In the present study only the electromagnetic (EM) equivalents (1 to 5 microTesla) of emotional words as well as intensity-matched $60 \mathrm{~Hz}$ sine wave fields were presented across the temporal lobes. The EM equivalents were produced by converting a digital audio signal into an electromagnetic field. We found that subjects selected significantly more words with the same pleasantness as the target word presented as an EM equivalent. We also found an enhancement of the effect for the presentation of unpleasant-active words. These results suggest that the electromagnetic component of the spoken word from electronics may mediate emotional information that affects decision-making.
\end{abstract}

Keywords: Word meaning, electromagnetic equivalents, alpha rhythms, decision-making.

\section{INTRODUCTION}

It has been demonstrated that experiences and responses can be evoked by means other than standard transduction pathways. Although brief exposures to weak radiofrequency electromagnetic fields [1] or aurora-related magnetic variations [2] pulsed within the audible range have produced reliable simple auditory sensations, more recent studies [3] have shown that pulsed magnetic fields applied over the left frontal lobe can increase the acceptance of false statements as true. The latter occurred without the subjects' "awareness". In addition, it has been reported that transcranial magnetic stimulation of the temporo-parietal junction alters an individual's ability to make morally-based decisions [4]. In the present experiment we replicated and extended a previous study [5] where subjects exposed to the electromagnetic field equivalent of different words selected other words with similar emotional meanings from a list of words with different meanings. These electromagnetic presentations were produced by converting a standard audio file, recorded with a computer, into an electromagnetic pattern by use of custom-built technology that converts a digital signal into an analogue equivalent.

Several experiments have shown that the application of simple, physiologically-patterned, approximately 1 microTesla (10 milligauss) magnetic fields across the temporal lobes produced experiences that involved reliable themes of subjective experiences [6]. This intensity is similar to what may be encountered through earphones or near a computer screen. Quantitative electroencephalography indicated chan-

*Address correspondence to this author at the Laurentian University, Department of Psychology, 935 Ramsey Lake Road, Sudbury, Ontario, P3E 2C6, Canada; Tel: 705-675-1151, ext. 4824;

E-mail: mpersinger@laurentian.ca ges in power within the right temporal-parietal and right frontal regions [7]. Preferential exposure over the right hemisphere has been associated with increased scores for hypnosis induction capacity or suggestibility $[8,9]$. This sensitivity of the right hemisphere to weak experimentally generated and natural geomagnetic fields has been reported frequently [10].

Words occupy two domains: categorical organization and emotional meaning. Considering the role of right hemispheric functions in the emotional processing of words [11] one might expect a detection of the affective characteristics of words when their electromagnetic rather than sonic patterns were presented. Healey and Persinger [5] exposed subjects for $30 \mathrm{~s}$ to the electromagnetic fields of digitized words obtained from the extremes of the four quadrants of the Whissell Dictionary of Affect [12,13]. It is composed of two dimensions: evaluation (unpleasant- pleasant) and activity (inactive-active). The subjects were then asked to select which word they "felt" was appropriate from a list of 5 words per trial. The selection lists included the target word (the one whose electromagnetic equivalent was being generated across the temporal lobes), 2 words from the orthogonally opposite quadrant relative to the target word, and 2 words which shared an emotional dimension with the target word (evaluation or activity). The subjects chose significantly more words that were from the same emotional quadrant as the target word.

Because the subjects chose words that were from the same emotional domain rather than the actual word, the authors concluded that inadvertent effects from bone conduction or artifact were unlikely. If decisions about which word to choose out of a limited sample of words with strong emotional meanings can be affected by the application of the electromagnetic equivalents of that word near the temporal 
lobes, then this effect may have social relevance. There is an increased propensity for people to listen to narratives and music with ear buds and earphones. In addition to the music and lyrics impinging as sonic energy, a magnetic field in the order of 1 microTesla is often produced within the electronics. It contains a shadow pattern of the sonic information that, unlike mechanical vibrations, could penetrate cerebral tissue.

\section{MATERIALS AND METHODS}

Ethics approval was obtained by the Department of Psychology Ethics Committee prior to any experimentation. Fifteen ( 8 male, 7 female) aged 20 to 25 years volunteered to be subjects from a first year psychology course. After administration and completion of consent forms, all subjects sat within a comfortable arm chair housed within a soundproof chamber. A Model P79 Electroencephalograph (EEG) was employed to monitor the bipolar activity over the occipital $(\mathrm{O} 1, \mathrm{O} 2)$, temporal $(\mathrm{T} 3, \mathrm{~T} 4)$ and frontal $(\mathrm{F} 7, \mathrm{~F} 8)$ lobes in accordance with the 10-20 International Standard of electrode placement. Two small boxes each containing 4 small solenoids (reed switches) was held by Velcro on each side of the subject's head at the level of the temporal lobes. The magnetic fields were generated between each pair (one on each side of the head) of solenoids. A Metex 3800 multimeter using a specialized probe indicated the field strengths were between 1 and 5 microT (10 to $50 \mathrm{mG}$ ) near the skull.

A total of 12 target words from each of the extreme quadrants of the Whissell dictionary were selected randomly. This means there were 3 words from each of the following quadrants: pleasant-passive (honour, beautiful, comfortable), pleasant-active (happy, marvelous, giving), unpleasant-passive (lonely, boredom, fowl), and unpleasant-active (anxiety, painful, anger). The number of syllables (hence word length) for each word was not significantly different across the four emotional quadrants. The original word records were completed with an Apple MacBook equipped with Audacity software and were sampled at $44 \mathrm{kHz}$. A program which utilized a DOS-based media player, Scoptrax, converted each of the audio files into electromagnetic equivalents. Hence the sonic components of the word were transformed into rows of numbers between 1 and 257. An example of the spatiotemporal organization of a word is depicted in Fig. (1).

\section{Comfortable}

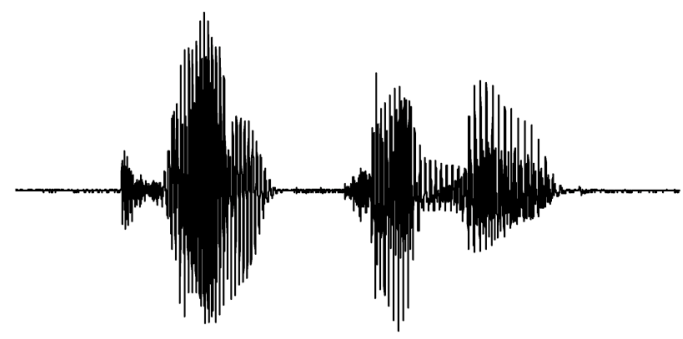

Fig. (1). A sample electromagnetic equivalent of a word pattern (comfortable) presented to the electromagnetic condition during the experiment.

The activation of each number for $1 \mathrm{msec}$ and the transformation of the numbers to an equivalent voltage between -
$5 \mathrm{~V}$ and $+5 \mathrm{~V}$ resulted in the electromagnetic equivalent of the word. The fidelity of the word was verified by placing a magnetic sensor coupled to an acoustic amplifier near the solenoids. The source numbers were generated from an IBM 286 computer with custom- constructed DACs (digital to analogue converters) that delivered the small changing voltages (and hence current) to the solenoids. There were two experimental groups. Eight subjects were exposed to the electromagnetic equivalents of the words. In order to control for non-specific effects from just the presence of a timevarying magnetic field seven subjects only received $60 \mathrm{~Hz}$ sine wave fields of comparable intensity and duration for each trial.

Each trial began with an instruction delivered through the closed chamber speaker to turn the page of a booklet and observe the list of 5 words. In addition to the word associated with the EM equivalent, there were 4 other words, 2 of which were similar in one emotional dimension (evaluation or activity) and 2 words originating from the orthogonally opposite quadrant (relative to the target word), all of which were selected from the Whissell dictionary. The electromagnetic (EM) equivalent of the word was presented for $60 \mathrm{~s}$. The duration of each EM word was 1-s and was presented 20 times with an inter-stimulus interval of $2 \mathrm{~s}$. After the application of the EM equivalent the subject was allowed $20 \mathrm{~s}$ to select a word that best matched the experiences of the previous $60 \mathrm{~s}$ before being instructed to turn to the next page. The next trial then began. Each trial was $60 \mathrm{~s}$ in duration and the total experimental time was $16 \mathrm{~min}$. The EEG was continuously recorded during the experiment.

Word selections were considered correct if they were either the target word or a word similar in an emotional dimension (pleasantness or activation). The EEG was scored for the proportion of time alpha rhythms or "spindles" (8 to $13 \mathrm{~Hz}$ ) that were displayed during each successive 4 min of the experiment.

\section{RESULTS}

A one-way analysis of variance revealed that the subjects exposed to the EM word equivalents were twice as likely $\left[\mathrm{F}(1,13)=6.35, \mathrm{p}<0.05\right.$; $\left.\mathrm{eta}^{2}=0.33\right]$ to select a word within the same pleasantness domain (unpleasant $v s$. pleasant) than the subjects exposed to the $60-\mathrm{Hz}$ magnetic field reference condition (Fig. 2A). To insure that this effect was not being

A

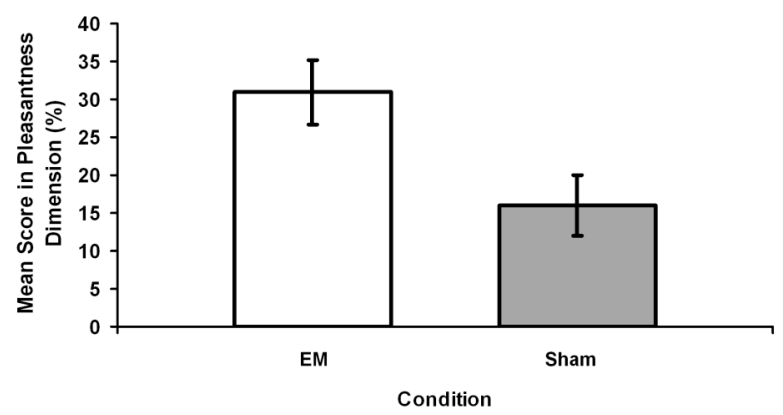

Fig. (2). A) Individuals exposed to the electromagnetic (EM) equivalents of words selected similar words in the pleasantness domain more frequently than the control group who were exposed to $60 \mathrm{~Hz}$ sine waves of matched intensity. (Vertical bars indicated standard errors of the mean). 
contaminated by indirect "hearing" of the target word, only target words were analyzed. A separate one-way analysis indicated that there was no significant difference $(F(1,13)$ $=0.00 ; p>0.05)$ between the groups (Fig. 2B).

B

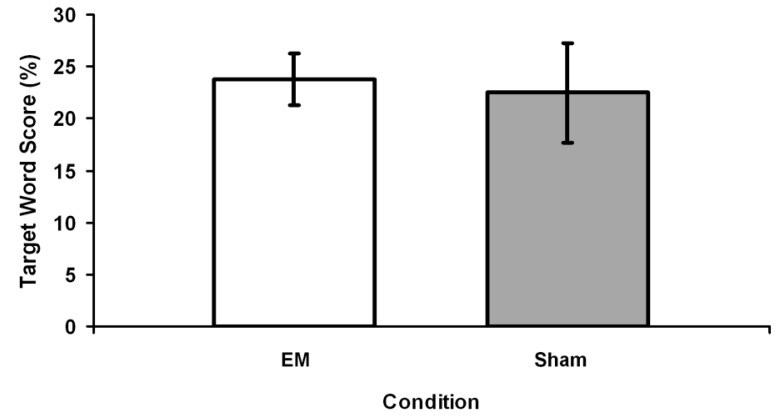

Fig. (2). B) There was no statistically significant difference in target word selection scores between the two conditions, thus minimizing the likelihood of indirect "hearing" from bone conduction $(\mathrm{EM}=$ electromagnetic $)$.

Four separate one-way analyses of variance were completed to discern if the subjects' accuracy varied for words within any one emotional quadrant. The results indicated that the EM group selected significantly more accurately $(\mathrm{F}(1,13)=15.87 ; \mathrm{p}<0.05$; omega-squared estimate $=0.55) \mathrm{EM}$ word patterns from the unpleasant-active quadrant compared to the $60-\mathrm{Hz}$ sham control group (Fig. 3). Group differences for the other three quadrants were not statistically significant. Ancillary analyses of the proportion of alpha rhythms displayed during the approximately $16 \mathrm{~min}$ experiment showed no significant changes over time between groups.

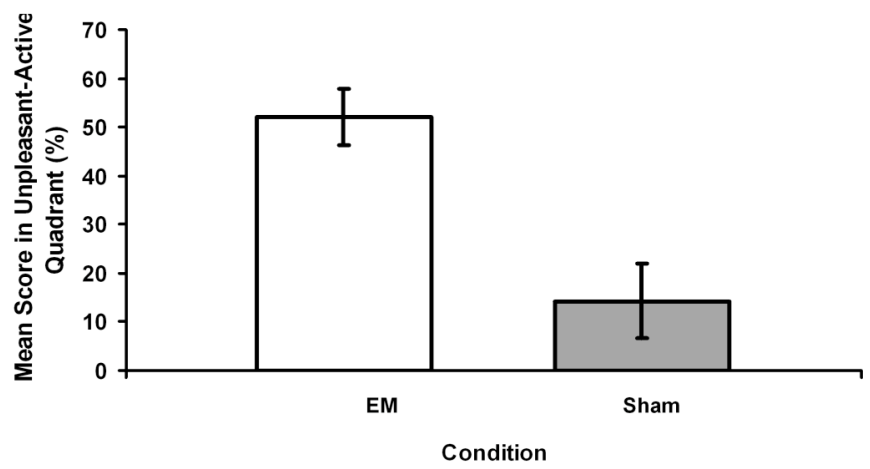

Fig. (3). The EM group selected significantly more accurately Unpleasant-Active words than individuals exposed to a sham-field.

\section{DISCUSSION}

The results of this experiment replicate and extend previous research [5]. The electromagnetic equivalent of specific emotive domains of words generated externally across the temporal lobes can affect people's choices. The group exposed to the EM patterns of 12 different words were twice as likely to select words from a list that were similar in emotional meaning to the target word compared to the group exposed to the intensity-matched, $60 \mathrm{~Hz}$ magnetic fields. The effects were specific to the information within the electromagnetic pattern of specific words and not the generalized response to the simple presence of an intensity-matched 60 $\mathrm{Hz}$ magnetic field. There is minimal information in $60 \mathrm{~Hz}$ sine-waves.
That this effect upon decision-making was not secondary to "inductive hearing" or artifact was indicated by the absence of differences between the two groups for actual target words. These results suggest that the brains were discerning the "emotional" components of the word equivalents.

The discernment of the "emotional" components of words has been considered a property of the right hemisphere [14]. In the present study words that shared unpleasantactive properties with the target words were more accurately discerned than words from other quadrants. This result is also consistent with the general function of the right hemisphere. Vigilance and unpleasant arousal are frequent correlates of excessive stimulation within this range.

If the "emotional" component of the spoken word can affect a person's decision when the electromagnetic (nonmechanical) equivalent is presented to the cerebrum, then complex-patterned magnetic fields may influence "unconscious" behavior more than realized. For decades increases in geomagnetic activity, which appears to influence (preferentially) right hemispheric functions [15] such as dreaming [16] have been associated with the more diffuse "affective" dysfunctions. Today's electronic society, whereby millions listen to music, lyrics, and narratives through earphones and earpieces directly over the temporal lobes, may be more subject to the unspoken meaning of the words than expected.

The technical process by which information is mediated through these devices to produce sound also produces concomitant magnetic fields within the same intensity range (1 microTesla) as the ones employed in this experiment. It may be relevant that the energy density between mechanical energies that produce hearing the values associated with a microTesla time-varying magnetic field are within the same order of magnitude as $40 \mathrm{db}$. For hearing this is equivalent to $20 \times 10^{-4} \mathrm{~N} / \mathrm{m}^{2}$ when applied within a volume (such as the air adjacent to the tympanic membrane) of $1 \mathrm{cc}\left(10^{-2}\right) \mathrm{m}^{3}$ and is about $2 \times 10^{-9} \mathrm{~J}$ (a nanoJoule).

According to Faraday's formula a 1 microTesla $\left(10^{-6} \mathrm{~T}\right), 1$ $\mathrm{kHz}\left(10^{3} \mathrm{~Hz}\right)$ magnetic field applied to $1 \mathrm{~cm}^{2}\left(10^{-4} \mathrm{~m}^{2}\right)$ crosssectional area produces a potential difference of $10^{-7} \mathrm{~V}$. Because a millimole concentration of one cubic centimeter of isotonic fluid within a neuronal tissue contains about $10^{-17}$ ions each displaying $1.6 \times 10^{-19} \mathrm{~A} \bullet \mathrm{s}$ (Coulombs), the total energy (voltage multiplied by charge) would be about $10^{-9} \mathrm{~J}$.

These estimates, which obviously require empirical verification by experimentation, suggest that variants of solenoids placed within the ears to hear music might also mediate local magnetic fields that extend into the adjacent temporal lobes. Although minuscule when compared to the energy generated within the brain volume (about 10 to 20 $\mathrm{J} / \mathrm{s}$ ) due to glucose metabolism, the $10^{-9} \mathrm{~J}$ order of magnitude is well with the range of energies $[17,18]$ for the sum of transients associated with action potentials $\left(10^{-20} \mathrm{~J}\right.$ per action potential) from the approximately $10^{10}$ to $10^{11}$ neurons within the cerebral cortices with average firing rates of about $10 \mathrm{~Hz}$.

\section{REFERENCES}

[1] Frey AH. Human auditory response to modulated electromagnetic energy. J App Physiol 1962; 17: 689-92

[2] Akasofu SI. Auroa Borelais: The amazing northern lights. Alaska Geographic 1979; 6: 1-95. 
[3] Ross ML, Koren SA, Persinger M. Physiologically patterned weak magnetic fields applied over left frontal lobe increase acceptance of false statements as true. Electromag Biol Med 2008; 27: 365-71.

[4] Young L, Comprodon JA, Hauser M, Pascual-Leone F, Sax R. Disruption of the right temporoparietal junction with transcranial magnetic stimulation reduces the role of beliefs in moral judgment. Proc Natl Acad Sci USA 2010; 107: 6753-8.

[5] Healey F, Persinger MA, Koren SA. Control of "choice" by application of the electromagnetic field equivalents of spoken words: mediation by emotional meaning rather than linguistic dimensions? Perceptual and Motor Skills 1997; 87: 1411-8.

[6] Persinger MA, Healey F. Experimental facilitation of the sensed presence: possible intercalation between the hemispheres induced by complex magnetic fields. J Nervous Mental Dis 2002; 190: 53341 .

[7] Booth JN, Koren SA, Persinger MA. Increased Theta Activity in Quantitative Electroencephalographic (QEEG) measurements during exposure to complex weak magnetic fields. Electromag Biol Med 2008; 27: 426-36.

[8] Tiller SA, Persinger MA. Enhanced hypnotizability by cerebrally applied magnetic fields depends upon the order of hemispheric presentation: An anistropic effect. Int J Neurosci 1994; 79: 157-63.

[9] Healey F, Persinger MA, Koren SA. Enhanced hypnotic suggestibility following application of burst-firing magnetic fields over the right temporoparietal lobes: a replication. Int $\mathbf{J}$ Neurosci 1996; 87: 201-7.

[10] Mulligan BP, Hunter MD, Persinger MA. Effects of geomagnetic activity and atmospheric power variations on quantitative measures of brain activity: Replication of the Azerbaijani studies. Adv Space Res 2010; 45: 940-8.

[11] Buchanan TW, Lutz K, Mirzazade S, et al. Recognition of emotional prosody and verbal components of spoken language: an fMRI study. Cognitive Brain Res 2000; 9: 227-38.

[12] Whissell CM, Povey G, Dewson M. Remembering "Mr. Smith:" memory for emotional connotations of personality describing words. J Soc Beh Person 1987; 2: 535-42.

[13] Whissell CM. The dictionary of affect in language. In: R. Plutchik, H. Kellerman (Eds.) Emotion: theory, research and experience. New York: Academic Press, 1989; pp. 113-31.

[14] Blonder LX, Bowers D, Heilman KM. The role of the right hemisphere in emotional communication. Brain 1991; 114: 111527.

[15] Booth JN, Koren SA, Persinger MA. Increased feelings of the sensed presence and increased geomagnetic activity at the time of the experience during exposures to transcerebral weak complex magnetic fields. Int J Neurosci 2005; 115: 1053-79.

[16] Krippner S, Persinger M. Evidence for enhanced congruence between dreams and distant target material during periods of decreased geomagnetic activity. J Sci Exp 1996; 10: 487-93.

[17] Persinger MA. $10^{-20}$ Joules as a neuromolecular quantum in medicinal chemistry: an alternative approach to myriad molecular pathways? Curr Med Chem 2010; 17: 3094-8.

[18] Persinger MA, Koren SA, Lafreniere GF. A neuroquantologic approach to how human thought might affect the universe. Neuro Quantol 2008; 6: 262-71.

(C) Saroka and Persinger; Licensee Bentham Open.

This is an open access article licensed under the terms of the Creative Commons Attribution Non-Commercial License (http://creativecommons.org/licenses/by$\mathrm{nc} / 3.0 /$ ), which permits unrestricted, non-commercial use, distribution and reproduction in any medium, provided the work is properly cited. 